\title{
On low-frequency dust-modes in a collisional and streaming dusty plasma with dust charge fluctuation
}

\author{
M. K. Islam, ${ }^{\text {a) }}$ Y. Nakashima, and K. Yatsu \\ Plasma Research Center, University of Tsukuba, Tsukuba, Ibaraki 305-8577, Japan \\ M. Salimullah \\ Department of Physics, Jahangirnagar University, Savar, Dhaka 1342, Bangladesh
}

(Received 22 August 2002; accepted 25 November 2002)

\begin{abstract}
Lighter particles streaming, dust charge fluctuation, and collisional effects on the dust-modes, viz., the dust-lower-hybrid (DLH) and dust-acoustic (DA) waves, have been examined analytically using fluid model. In the absence of dust charge fluctuation and collisional effects, two-stream instability of these modes occurs when the ion streaming velocity exceeds the parallel phase velocity of the respective mode. Moreover, if either ion or electron streaming velocity exceeds the wave parallel phase velocity, then the collisions of ions or electrons with neutral gas atoms/molecules give destabilizing effect on the dust-modes provided the dust charge fluctuation is negligible. Dust charge fluctuation gives the damping of either of these modes. It is found that charge fluctuation and collisional effects on the DA-mode are greater than those of the DLH-mode. Hence, the DLH-mode is more stable compared to the DA-mode. (C) 2003 American Institute of Physics.
\end{abstract}

[DOI: $10.1063 / 1.1539474$ ]

\section{INTRODUCTION}

Since the discovery of low-frequency dust-acoustic (DA) and dust-ion-acoustic (DIA) waves in unmagnetized dusty plasmas, ${ }^{1,2}$ dusty plasmas are being extensively studied. Highly charged and relatively massive - compared to ionsdust grains exist both in natural and laboratory plasmas. The presence of dust in plasma can modify the existing plasma modes or may introduce new eigenmodes. ${ }^{3}$ Theoretical and experimental research in dusty plasma has expanded into a wider range of problems, including studies of collective processes, i.e., waves and instabilities. Experiments in the laboratory have verified the theoretical predictions of the DA waves, ${ }^{4,5}$ the DIA waves, ${ }^{6,7}$ and the dust-lattice (DL) wave. ${ }^{8}$ Merlino et al. experimentally studied the properties of DIA waves and electrostatic dust-ion-cyclotron (EDIC) waves and have shown that these modes are more easily excited in a plasma containing negatively charged dust. ${ }^{9}$ They also observed the DA-mode and found a good agreement of the mode properties with that predicted by fluid theory. However, magnetized dusty plasmas obviously support more additional low-frequency electrostatic dust-modes, such as the dust-lower-hybrid (DLH) mode of frequency below the ioncyclotron frequency. ${ }^{10,11}$ Recently, D'Angelo has explained theoretically the excitation of the DLH-mode in a laboratory and shown that the DLH-mode can be excited easily, compared to the electrostatic dust-cyclotron (EDC) mode, in the presence of zeroth-order electric field. ${ }^{12}$

In high-density cold plasma, the DLH-mode arises due to the dynamics of negatively charged unmagnetized dust grains and the magnetized ions. If the electrons are consid-

a) Permanent address: Institute of Nuclear Science and Technology, Atomic Energy Research Establishment, Ganakbari, Savar, Dhaka 1349 (G.P.O. Box 3787, Dhaka 1000), Bangladesh; electronic mail: khairulislam@yahoo.com ered as a hot Boltzmann gas at temperature $T_{e}$ in low-density magnetized dusty plasma, then the DA-mode may be excited, which propagates nearly perpendicularly to the external static magnetic field, i.e., in the direction of the DLH-mode. ${ }^{13}$ It was shown that this DA-mode is damped rapidly, compared to the DLH-mode, through dust charge fluctuation and Landau damping processes. This study was carried out using the Vlasov-Poisson model in the collisionless limit. Moreover, the streaming effect on the mode properties was not studied sufficiently.

Due to the heavier mass of the dust compared to ions, dust dynamics introduce low-frequency dust-modes below the ion-cyclotron frequency in dusty plasmas. In this lowfrequency limit, collision of the charged particles with the neutrals may play important roles in the properties of dustmodes. Moreover, in real plasma, the time dependent variation of dust charge occurs when the conditions in the plasma near the dust grain are changed due to a variety of reasons, such as the wave motion. Streaming of plasma particles can occur due to the presence of an ambient electric field. In a laboratory, the electric field may be generated in the sheath region on account of the high mobility of the electrons. In the space plasma system, this electric field may be established due to inhomogeneous distribution of charge carriers. Therefore, theoretical study is carried out on the dust-modes (viz., DLH, DA) using a fluid model in a collisional, streaming magnetized dusty plasma in the presence of zeroth-order electric field including dust charge fluctuation. Two-stream instability of the dust-modes due to the streaming of lighter particles of the plasma has been examined analytically. Dust charge fluctuation and collisional effects on the dust-modes are studied. Comparison of the dust charge fluctuation and collision effects between these modes is also carried out. In this study, it is considered that the dust grain size $\left(a_{d}\right)$ is much smaller compared to the average Larmor radius $\left(r_{j}\right)$ of 
the plasma particles, i.e., $r_{j}>a_{d}$, where $j$ stands for the plasma species. In this limit the charging equation of the dust grain in unmagnetized plasma can be applied to the magnetized plasma.

The manuscript is organized as follows: In Sec. II, fluid theory for dust-modes is given. Dispersion relations of the electrostatic dust-modes and low-frequency electrostatic dust-modes are given respectively, in Secs. II A and II B. In Sec. III, two-stream instability of the low-frequency electrostatic dust-modes is discussed. Damping and instability of the DLH- and DA-modes are given in Sec. IV. Comparison of dust charge fluctuation and collisional effects between the DLH- and DA-modes is also given in this section. Finally, in Sec. V, discussions and conclusions of our results are given.

\section{THEORY}

\section{A. General dispersion relation of dust-modes}

We have considered a homogeneous and uniform magnetized dusty plasma consisting of electrons, ions, negatively charged dust grains and neutrals. The zeroth-order electric $\left(\mathbf{E}_{o}\right)$ and magnetic (B) fields are in the $z$-direction. The charged plasma particles have zeroth-order constant velocities, $v_{j o}$, due to the electric field $\mathbf{E}_{o}$. The neutral gas is taken to be at rest. We consider the zeroth-order streaming of the electrons and ions relative to the dust grains in the $z$-direction. The dust charge is considered as negative, i.e., $Q_{d}=-Z_{d} e$, with $e$ being the magnitude of electron charge. Let us consider an electrostatic wave propagating obliquely to the external magnetic field with propagation vector $(\mathbf{K})$ lying in the $x z$-plane. Due to the presence of this mode $(\omega, \mathbf{K})$, the dust will acquire a perturbed charge, $Q_{d 1}$. The theory of oblique propagation of the electrostatic wave in the described dusty plasma is given below:

Continuity and momentum equations for different species of the dusty plasma are taken as follows, respectively:

$$
\begin{aligned}
& \frac{\partial n_{+}}{\partial t}+\nabla \cdot\left(n_{+} \mathbf{v}_{+}\right)=0, \\
& \frac{\partial n_{e}}{\partial t}+\nabla \cdot\left(n_{e} \mathbf{v}_{e}\right)=0, \\
& \frac{\partial n_{d}}{\partial t}+\nabla \cdot\left(n_{d} \mathbf{v}_{d}\right)=0,
\end{aligned}
$$

and

$$
\begin{aligned}
& n_{+} m_{+} \frac{\partial \mathbf{v}_{+}}{\partial t}+n_{+} m_{+} \mathbf{v}_{+} \cdot \nabla \mathbf{v}_{+}+k T_{+} \nabla n_{+}-e n_{+} \mathbf{E} \\
& \quad-e n_{+} \mathbf{v}_{+} \times \mathbf{B}=-\nu_{+} n_{+} m_{+} \mathbf{v}_{+}, \\
& k T_{e} \nabla n_{e}+e n_{e} \mathbf{E}+e n_{e} \mathbf{v}_{e} \times \mathbf{B}=-\nu_{e} n_{e} m_{e} \mathbf{v}_{e}, \\
& n_{d} m_{d} \frac{\partial \mathbf{v}_{d}}{\partial t}+n_{d} m_{d} \mathbf{v}_{d} . \nabla \mathbf{v}_{d}+k T_{d} \nabla n_{d}+e Z_{d} n_{d} \mathbf{E}+e Z_{d} n_{d} \mathbf{v}_{d} \times \mathbf{B} \\
& \quad=-\nu_{d} n_{d} m_{d} \mathbf{v}_{d} .
\end{aligned}
$$

The subscripts $d,+$, and $e$ denote respectively, the dust grain, ion and electron quantities. All the remaining symbols have their usual meaning.

The continuity and momentum Eqs. (1)-(6) will be coupled to the following set of equations, namely: Poisson's equation

$$
-\varepsilon_{o} \nabla^{2} \phi=e\left[n_{+}-Z_{d} n_{d}-n_{e}\right],
$$

quasi-neutrality equation

$$
n_{+}=n_{e}+Z_{d} n_{d},
$$

and basic dust charging equation

$$
\frac{d Q_{d}}{d t}=I_{e}+I_{i},
$$

where $I_{e}$ and $I_{i}$ are, respectively, the electron and ion currents collected by the dust grains.

The average electron and ion currents involved in the basic dust charging Eq. (9) for cold plasma can be derived from the following equation:

$$
I_{j}=\left(e_{j} n_{j} v_{j}\right) \pi a_{d}^{2}\left[1-\frac{2 e_{j}\left(\phi_{p}-\phi_{d}\right)}{m_{j} v_{j}^{2}}\right],
$$

where $a_{d}, \phi_{p}$, and $\phi_{d}$ are the dust radius, bulk plasma potential, and dust grain surface potential, respectively.

The plasma is steady and uniform $(\partial / \partial t=0=\partial / \partial x$ $=\partial / \partial y)$ at zeroth-order state, where the electric field, $\mathbf{E}_{o}$, is constant and the velocities of the three charged components are also constant. We find the zeroth-order velocities of the charged plasma particles:

$$
\begin{aligned}
& e E_{o}=\nu_{+} m_{+} v_{+o}, \\
& e E_{o}=-\nu_{e} m_{e} v_{e o}, \\
& e Z_{d o} E_{o}=-\nu_{d} m_{d} v_{d o} .
\end{aligned}
$$

The quasi-neutral condition at zeroth-order state becomes

$$
n_{+o}=n_{e o}+Z_{d o} n_{d o} .
$$

Equations (1)-(10) are linearized and the first-order quantities are taken to have the space and time dependence $e^{i\left(K_{x} x+K_{z} z-\omega t\right)}$. We get

$\frac{\partial n_{+1}}{\partial t}+n_{+o}\left(\nabla \cdot \mathbf{v}_{+1}\right)+\left(\mathbf{v}_{+o} \cdot \nabla\right) n_{+1}=0$,

$\frac{\partial n_{e 1}}{\partial t}+n_{e o}\left(\nabla \cdot \mathbf{v}_{e 1}\right)+\left(\mathbf{v}_{e o} \cdot \nabla\right) n_{e 1}=0$

$\frac{\partial n_{d 1}}{\partial t}+n_{d o}\left(\nabla \cdot \mathbf{v}_{d 1}\right)+\left(\mathbf{v}_{d o} \cdot \nabla\right) n_{d 1}=0$,

$m_{+} n_{+o}\left[\frac{\partial \mathbf{v}_{+1}}{\partial t}+\left(\mathbf{v}_{+o} \cdot \nabla\right) v_{+1}\right]+k T_{+} \nabla n_{+1}-e n_{+o} \mathbf{E}_{1}$

$$
-e n_{+1} \mathbf{E}_{0}-e n_{+o} \mathbf{v}_{+1} \times \mathbf{B}_{o}
$$$$
=-\nu_{+} m_{+} n_{+o} \mathbf{v}_{+1}-\nu_{+} m_{+} n_{+1} \mathbf{v}_{+o},
$$

$k T_{e} \nabla n_{e}+e n_{e o} \mathbf{E}_{1}+e n_{e 1} \mathbf{E}_{o}+e n_{e o} \mathbf{v}_{e 1} \times \mathbf{B}_{o}$

$=-\nu_{e} m_{e} n_{e o} \mathbf{v}_{e 1}-\nu_{e} m_{e} n_{e 1} \mathbf{v}_{e o}$, 


$$
\begin{aligned}
& m_{d} n_{d o}\left[\frac{\partial \mathbf{v}_{d 1}}{\partial t}+\left(\mathbf{v}_{d o} . \nabla\right) \mathbf{v}_{d 1}\right]+k T_{d} \nabla n_{d 1}+e Z_{d} n_{d o} \mathbf{E}_{1} \\
& +e Z_{d} n_{d 1} \mathbf{E}_{o}+e n_{d o} \mathbf{v}_{d 1} \times \mathbf{B}_{o} \\
& =-\nu_{d} m_{d} n_{d o} \mathbf{v}_{d 1}-\nu_{d} m_{d} n_{d 1} \mathbf{v}_{d o}, \\
& -\varepsilon_{o} \nabla^{2} \phi=e\left[n_{+1}-Z_{d o} n_{d 1}+\frac{Q_{d 1}}{e} n_{d o}-n_{e 1}\right], \\
& \frac{d Q_{d 1}}{d t}=I_{e 1}+I_{i 1},
\end{aligned}
$$

where

$$
I_{j 1}=e_{j} \pi a_{d}^{2}\left(n_{j o} v_{j 1}+n_{j 1} v_{j o}\right)\left(1+\frac{2 e_{j} \phi_{d 0}}{m_{j} v_{j 0}^{2}}\right) .
$$

We have considered that $\nabla n_{d o}=\nabla n_{+o}=\nabla n_{e o}=\phi_{p o}=0$ and because of zeroth-order constraints $\left|I_{e o}\right|=\left|I_{i o}\right|$.

Let us define the Doppler shifted frequency due to the streaming of the charged particles as $\Omega_{+}=\omega-K_{z} v_{+o}, \Omega_{d}$ $=\omega-K_{z} v_{d o}$, and $\Omega_{e}=\omega-K_{z} v_{e o}$, where $v_{e o}, v_{+o} \gg v_{d o}$. From the linearized continuity [Eq. (15)] and momentum [Eq. (18)] equations for the ions, we obtain, respectively,

$$
\frac{n_{+1}}{n_{+o}} \Omega_{+}=K_{x} v_{+1 x}+K_{z} v_{+1 z}
$$

and

$$
\begin{aligned}
& x \text {-component: }\left(\Omega_{+}+i \nu_{+}\right) v_{+1 x}-i \omega_{c+} v_{+1 y} \\
& \quad-K_{x} C_{+}^{2} \varepsilon_{+}-K_{x} e / m_{+} \phi_{1}=0, \\
& y \text {-component: }\left(\Omega_{+}+i \nu_{+}\right) v_{+1 y}+i \omega_{c+} v_{+1 x}=0, \\
& z \text {-component: }\left(\Omega_{+}+i \nu_{+}\right) v_{+1 z}-K_{z} C_{+}^{2} \varepsilon_{+}-K_{z} e / m_{+} \phi_{1}=0,
\end{aligned}
$$

where the ion thermal velocity $C_{+}^{2}=k T_{+} / m_{+}$and ion cyclotron frequency $\omega_{c+}=e B_{z} / m_{+}$.

From Eqs. (24) and (25), we get

$$
n_{+1}=\frac{e n_{+o} R \phi_{1}}{m_{+}\left(\Omega_{+}-C_{+}^{2} R\right)} .
$$

In a similar manner, from the linearized continuity and momentum equations for the electrons and dusts we obtain

$$
n_{e 1}=\frac{i e n_{e o} F \phi_{1}}{m_{e}\left(\Omega_{e}+i C_{e}^{2} F\right)}
$$

and

$$
n_{d 1}=-\frac{e Z_{d o} n_{d o} L \phi_{1}}{m_{d}\left(\Omega_{d}-C_{d}^{2} L\right)},
$$

where

$$
\begin{aligned}
& R=\frac{\left(K_{x}^{2}+K_{z}^{2}\right)\left(\Omega_{+}+i \nu_{+}\right)^{2}-K_{z}^{2} \omega_{+c}^{2}}{\left[\left(\Omega_{+}+i \nu_{+}\right)^{2}-\omega_{c+}^{2}\right]\left(\Omega_{+}+i \nu_{+}\right)}, \\
& F=\frac{\left(K_{x}^{2}+K_{z}^{2}\right) \nu_{e}^{2}+K_{z}^{2} \omega_{c e}^{2}}{\left(\nu_{e}^{2}+\omega_{c e}^{2}\right) \nu_{e}},
\end{aligned}
$$

$$
L=\frac{\left(K_{x}^{2}+K_{z}^{2}\right)\left(\Omega_{d}+i \nu_{d}\right)^{2}-K_{z}^{2} \omega_{c d}^{2}}{\left[\left(\Omega_{d}+i \nu_{d}\right)^{2}-\omega_{c d}^{2}\right]\left(\Omega_{d}+i \nu_{d}\right)} .
$$

The perturbed velocities of the species, $j$, in the case of an unmagnetized dusty plasma can be obtained from Eqs. (1)(3) and are given by

$$
v_{j 1}=\frac{n_{j 1}}{n_{j o}} \frac{\Omega_{j}}{K} .
$$

Using the perturbed quantities from Eq. (30), we get the perturbed dust charge, $Q_{d 1}$, from Eq. (22) as

$$
Q_{d 1}=i\left|I_{e o}\right| \frac{1}{K v_{+o}}\left[\frac{n_{+1}}{n_{+o}}-\frac{n_{e 1}}{n_{e o}} \frac{v_{+o}}{v_{e o}}\right] .
$$

Using the value of $Q_{d 1}$ from Eq. (31), we get the linearized Poisson's Eq. (21) as

$$
\begin{aligned}
\phi_{1}= & \frac{e}{\varepsilon_{o} K^{2}}\left[n_{+1}\left\{1+\frac{i \beta}{K v_{+o}} \frac{n_{e o}}{n_{+o}}\right\}-n_{e 1}\left\{1+\frac{i \beta}{K v_{e o}}\right\}\right. \\
& \left.-Z_{d o} n_{d 1}\right],
\end{aligned}
$$

where $\beta=\left(\left|I_{e o}\right| / e.\right)\left(n_{d o} / n_{e o}\right)=10^{-1} \pi a_{d}^{2} n_{d o} C_{e}$ and the $i \beta$ terms are arising through coupling to dust charge fluctuations which result as a response to collective plasma perturbation.

The dispersion relation for the electrostatic dust-modes is then obtained from Eq. (32) by putting the value of $n_{+1}$, $n_{e 1}$, and $n_{d 1}$ from Eqs. (26), (27), and(28), respectively. The dispersion relation is then given by

$$
\begin{aligned}
1= & \frac{\omega_{p+}^{2} R}{K^{2}\left(\Omega_{+}-C_{+}^{2} R\right)}\left\{1+\frac{i \beta}{K v_{+o}} \frac{n_{e o}}{n_{+o}}\right\} \\
& -\frac{i \omega_{p e}^{2} F}{K^{2}\left(\Omega_{e}+i C_{e}^{2} F\right)}\left\{1+\frac{i \beta}{K v_{e o}}\right\}+\frac{\omega_{p d}^{2} L}{K^{2}\left(\Omega_{d}-C_{d}^{2} L\right)},
\end{aligned}
$$

where the plasma frequency of the species $j$ is $\omega_{p j}^{2}$ $=n_{j} e_{j}^{2} / \varepsilon_{o} m_{j}$. The dispersion relation of Eq. (33) of the electrostatic dust-modes in collisional, streaming magnetized dusty plasmas is the basic one for the analysis of lowfrequency electrostatic dust-modes.

\section{B. Dispersion relation of low-frequency dust-modes}

Analogous to the dust-acoustic wave, we consider the presence of a low-frequency electrostatic dust-mode below the ion-cyclotron frequency propagating nearly perpendicularly $\left(K_{x}^{2} \gg K_{z}^{2}\right)$ to the external static magnetic field in the magnetized dusty plasma where the dynamics of the dust particles may play an important role. In this case, we assume that the dust-mode under consideration satisfies the following conditions:

$$
\omega_{c d} \ll \omega \ll \omega_{c i} \ll \omega_{c e} ; \quad K C_{d}, K_{z} C_{+} \ll \omega \ll K_{z} C_{e} .
$$

Under these conditions, the highly charged and massive dust grains can be taken to be cold $\left(C_{d}=0\right)$ and unmagnetized $\left(\omega_{c d}=0\right)$. Ions are cold $\left(C_{+}=0\right)$, but strongly magnetized. Electrons form a hot Boltzmann gas at temperature $T_{e}$. As 
$m_{d} \gg m_{i}, m_{e}$, collision of dusts with neutrals may be neglected $\left(\nu_{d}=0\right)$. Under these conditions Eq. (33) gives the dispersion relation for our required dust-modes:

$$
\omega^{2}=\omega_{D M}^{2}-i \beta^{\prime}-i \nu_{+}^{\prime}-i \nu_{e}^{\prime}
$$

where

$$
\begin{aligned}
& \omega_{D M}^{2}=\frac{\left(K_{z}^{2} \omega_{p+}^{2} / K^{2} \Omega_{+}^{2}\right) \omega^{2}+\left(\omega_{p d}^{2} / \Omega_{d}^{2}\right) \omega^{2}}{1+1 / K^{2} \lambda_{D e}^{2}+K_{x}^{2} \omega_{p+}^{2} / K^{2} \omega_{c+}^{2}}, \\
& \beta^{\prime}=\beta \frac{\left(K_{x}^{2} n_{e o} \omega_{p+}^{2} / K^{3} v_{+o} n_{+o} \omega_{c+}^{2}\right) \omega^{2}+\left(1 / K^{3} v_{e o} \lambda_{D e}^{2}\right) \omega^{2}}{1+\left(1 / K^{2} \lambda_{D e}^{2}\right)+\left(K_{x}^{2} \omega_{p+}^{2} / K^{2} \omega_{c+}^{2}\right)},
\end{aligned}
$$

$\nu_{+}^{\prime}=\nu_{+} \frac{\left(K_{x}^{2} \omega_{p+}^{2} / K^{2} \omega_{c+}^{2} \Omega_{+}\right) \omega^{2}}{1+1 / K^{2} \lambda_{D e}^{2}+K_{x}^{2} \omega_{p+}^{2} / K^{2} \omega_{c+}^{2}}$,

and

$$
\nu_{e}^{\prime}=\nu_{e} \frac{\left(\Omega_{e} / K_{z}^{2} K^{2} \lambda_{D e}^{2} C_{e}^{2}\right) \omega^{2}}{1+1 / K^{2} \lambda_{D e}^{2}+K_{x}^{2} \omega_{p+}^{2} / K^{2} \omega_{c+}^{2}} .
$$

The dispersion relation of Eq. (34) has been used for the analysis of the properties and instabilities of the electrostatic dust-modes below the ion-cyclotron frequency in the following sections.

\section{TWO-STREAM INSTABILITY}

To discuss the two-stream instability of these dustmodes, we consider $\beta^{\prime}=\nu_{+}^{\prime}=\nu_{e}^{\prime}=0$. In this case Eq. (34) becomes

$$
\omega^{4}-B \omega^{3}+C \omega^{2}+D \omega-E=0,
$$

where $B=2 K_{z} v_{+o}, C=K_{z}^{2} v_{+o}^{2}-\omega^{2}, E=K_{z}^{2} v_{+o}^{2} \omega_{p d}^{2} / A, A$ $=1+1 / K^{2} \lambda_{D e}^{2}+K_{x}^{2} \omega_{p+}^{2} / K^{2} \omega_{c+}^{2}, \quad$ and $\quad \omega^{2}=(1$ $\left.+K_{z}^{2} \omega_{p+}^{2} / K^{2} \omega_{p d}^{2}\right) \omega_{p d}^{2} / A$. For simplicity of understanding, we have considered $v_{d o}=0$, because of the heavy mass of dust grains compared to ions and electrons.

If $K_{z} v_{+o}>\omega$, then $C>B$ and $E>D$. Thus, Eq. (39) reduces to quadratic equation as follows:

$$
\omega^{4}+C \omega^{2}-E=0
$$

Two roots of Eq. (40) are as

$$
\begin{aligned}
& \omega_{1}^{2}=-\frac{1}{2} C+\left(\frac{1}{4} C+E\right)^{1 / 2}, \\
& \omega_{2}^{2}=-\frac{1}{2} C-\left(\frac{1}{4} C+E\right)^{1 / 2} .
\end{aligned}
$$

The root $\omega_{1}^{2}$ is positive real quantities and therefore there can be no temporal growth or decay of the amplitude of dustmodes. On the other hand, $\omega_{2}^{2}$ is a negative real quantity. Therefore, $\omega_{2}$ has two imaginary values (one positive and one negative). The positive imaginary value of $\omega_{2}$ corresponds to an unstable mode and the growth rate of the mode is given by

$$
\begin{aligned}
\gamma_{2}= & {\left[\frac{1}{2} C+\left(\frac{1}{4} C+E\right)^{1 / 2}\right]^{1 / 2} } \\
= & {\left[\frac{1}{2}\left(K_{z}^{2} v_{+o}^{2}-\omega^{2}\right)+\left(\frac{1}{4}\left(K_{z}^{2} v_{+o}^{2}-\omega^{2}\right)\right.\right.} \\
& \left.\left.+\frac{K_{z}^{2} v_{+o}^{2} \omega_{p d}^{2}}{1+1 / K^{2} \lambda_{D e}^{2}+K_{x}^{2} \omega_{p+}^{2} / K^{2} \omega_{c+}^{2}}\right)^{1 / 2}\right]^{1 / 2} .
\end{aligned}
$$

On the other hand, if $\omega>K_{z} v_{+o}$, then $C$ becomes negative and $C>E$. Thus, Eq. (39) reduces to $\omega^{2}=C$, and there is no instability.

Thus, the two-stream instability of the electrostatic dustmodes below the ion-cyclotron frequency in streaming dusty plasmas, which propagate nearly perpendicular to the external magnetic field, arises only when the ion streaming velocity exceeds the parallel phase velocity of the wave.

\section{DLH- AND DA-MODES}

\section{A. Damping and instability}

For simplicity of the analysis of low-frequency, lowphase velocity electrostatic dust-modes in the magnetized dusty plasma, two important cases of dusty plasma are considered: case A: high-density dusty plasma and case B: lowdensity dusty plasma. In either of the cases, we have neglected the zeroth-order velocity of the massive dust grains, i.e., $v_{d o}=0$.

\section{Case A: High-density dusty plasma, i.e.,} $\omega_{p+}^{2} / \omega_{c+}^{2}>1 / K^{2} \lambda_{D e}^{2}$

In this case, the dispersion relation of dust-lower-hybrid wave can be obtained from Eq. (34), which is as follows:

$$
\begin{aligned}
\omega^{2}= & \omega_{d l h}^{2}\left[1+\frac{K_{z}^{2}}{K^{2}} \frac{\omega_{p+}^{2} \omega^{2}}{\omega_{p d}^{2} \Omega_{+}^{2}}\right]-i \beta \frac{n_{e o} \omega^{2}}{K v_{+o} n_{+o}}-i \nu_{+} \frac{\omega^{2}}{\Omega_{+}} \\
& -i \nu_{e} \frac{\Omega_{e} \omega_{c+}^{2} \omega^{2}}{K_{z}^{2} K^{2} C_{e}^{2} \lambda_{D e}^{2} \omega_{p+}^{2}}
\end{aligned}
$$

where $\omega_{d l h}^{2}=\omega_{p d}^{2} \omega_{c+}^{2} / \omega_{p+}^{2}=\omega_{c+}^{2} \omega_{c d}^{2}\left(Z_{d o} n_{d o} / n_{+o}\right)$.

The natural DLH-mode can be obtained from the above equation when $v_{+o}=\beta=\nu_{+}^{\prime}=\nu_{e}^{\prime}=0$. When $\beta=\nu_{+}^{\prime}=\nu_{e}^{\prime}$ $=0$, but $v_{+o} \neq 0$, then the two-stream instability of the mode will occur provided $\omega>K_{z} v_{+o}$ and the growth rate of the instability can be obtained from Eq. (43). The second term on the right hand side (RHS) of Eq. (44) presents the dust charge fluctuation of the DLH-mode, which gives the damping of the mode. From the third and fourth terms of the RHS of Eq. (44), it is seen that if either ion or electron streaming velocity exceeds the wave parallel phase velocity, then the collisions of ions or electrons with neutral gas atoms/ molecules gives destabilizing effect provided the dust charge fluctuation is negligible.

2. Case B: Low-density dusty plasma, i.e., $1 / K^{2} \lambda_{D e}^{2}$ $>\omega_{p+}^{2} / \omega_{c+}^{2}$

In this case, the dispersion relation of dust-acoustic waves can be obtained from Eq. (34), which is as follows: 


$$
\begin{aligned}
\omega^{2}= & K^{2} \omega_{D A}^{2}\left[1+\frac{K_{z}^{2}}{K^{2}} \frac{\omega_{p+}^{2} \omega^{2}}{\omega_{p d}^{2} \Omega_{+}^{2}}\right]-i \beta \frac{\omega^{2}}{K v_{e o}} \\
& -i \nu_{+} \frac{\omega_{p+}^{2} K^{2} \lambda_{D e}^{2} \omega^{2}}{\omega_{c+}^{2} \Omega_{+}}-i \nu_{e} \frac{\Omega_{e} \omega^{2}}{K_{z}^{2} C_{e}^{2}},
\end{aligned}
$$

where $\omega_{D A}^{2}=C_{e}^{2} \omega_{p d}^{2} / 2 \omega_{p e}^{2}$.

The natural DA-mode can be obtained from the above equation when $v_{j o}=\beta=\nu_{+}^{\prime}=\nu_{e}^{\prime}=0$. When $\beta=\nu_{+}^{\prime}=\nu_{e}^{\prime}=0$, but $v_{+o} \neq 0$, then the two-stream instability of the mode will occur provided $\omega>K_{z} v_{+o}$ and the growth rate of the instability can be obtained from Eq. (43). The second term on the RHS of Eq. (45) presents the dust charge fluctuation of the DA-mode, which gives the damping of the mode. From the third and fourth terms of the RHS of Eq. (45), it is seen that if either ion or electron streaming velocity exceeds the wave parallel phase velocity, then the collisions of ions or electrons with neutral gas atoms/molecules gives destabilizing effect provided the dust charge fluctuation is negligible.

\section{B. Comparison of dust charge fluctuation and collision effects between the dust-modes}

The ratios of dust charge fluctuation and collisional effects between the DLH- and DA-modes can be obtained from Eqs. (44) and (45) and are given by

$$
\begin{aligned}
& \frac{\beta_{D A}^{\prime}}{\beta_{d l h}^{\prime}}=\frac{n_{+o}}{n_{e o}} \frac{v_{+o}}{v_{e o}}, \\
& \frac{\nu_{+D A}}{\nu_{+d l h}}=\frac{\nu_{e D A}}{\nu_{e d l h}}=\frac{\omega_{p+}^{2}}{\omega_{c+}^{2}} K^{2} \lambda_{D e}^{2} .
\end{aligned}
$$

For negatively charged dust grains, $n_{+_{o}} / n_{e o} \gg 1$. If $v_{+o} / v_{e o} \approx 1$ and $K^{2} \lambda_{D e}^{2} \approx 1$, then it is found from Eqs. (46) and (47) that dust charge fluctuation and collisional effects on the DA-mode are much greater than those of the DLHmode. Hence, the DLH-mode is stable compared to the DAmode.

\section{DISCUSSIONS AND CONCLUSIONS}

Using the fluid model, an investigation of the properties and excitation of low-frequency and low-phase velocity electrostatic waves below the ion cyclotron frequency in a magnetized dusty plasma with lighter particles streaming, dust charge fluctuation, and collisional effects has been performed analytically. In high-density cold magnetized dusty plasma, the DLH-mode arises due to the dynamics of negatively charged unmagnetized dust grains and the magnetized ions [cf. Eq. (44)]. If the electrons are considered as hot Boltzmann gas at temperature $T_{e}$ in low-density magnetized dusty plasma, then DA-mode is obtained, which propagates nearly perpendicular to the external static magnetic field [cf. Eq. (45)].
It is found that only the streaming velocity of ions is coupled directly with the DLH and DA modes. In the absence of dust charge fluctuation and collisional effects, twostream instability of the DLH and DA modes occurs when the ion streaming velocity exceeds the parallel phase velocity of the respective mode. It is also found that the streaming energies of ions and electrons are coupled with the mode through the collisions of the particles with the neutrals. Through this coupling, a low-phase velocity wave can take the particle streaming energy. Due to the gain of energy, amplitude of the mode will grow. Therefore, the collision of the streaming particles with the neutrals can destabilize the mode. It is found that if either ion or electron streaming velocity exceeds the wave parallel phase velocity, then the collisions of ions or electrons with neutral gas atoms/ molecules give destabilizing effect on the DLH and DA modes provided the dust charge fluctuation is negligible. Dust charge fluctuation gives the damping of either of these modes. For negatively charged dust grains $n_{+o} / n_{e o} \ll 1$. Therefore, if $\mathbf{v}_{+o} / \mathbf{v}_{e o} \approx 1$ and $\mathbf{K}^{2} \lambda_{D e}^{2} \approx 1$, then it is found that the charge fluctuation and collisional effects on the DAmode are much greater than those of the DLH-mode. Hence, the DLH-mode is more stable compared to the DA-mode.

In conclusion, it is stressed that the results of the present investigation should be useful in understanding the various properties and effects of extremely low-frequency electrostatic dust-modes in a laboratory dusty magnetoplasmas.

\section{ACKNOWLEDGMENTS}

It is a pleasure to acknowledge the encouragement of the members of Plasma Research Center, University of Tsukuba.

The financial support of the University of Tsukuba is thankfully acknowledged.

${ }^{1}$ N. N. Rao, P. K. Shukla, and M. Y. Yu, Planet. Space Sci. 38, 543 (1990).

${ }^{2}$ P. K. Shukla and V. P. Silin, Phys. Scr. 45, 508 (1992).

${ }^{3}$ P. K. Shukla, Phys. Plasmas 8, 1791 (2001).

${ }^{4}$ J. Chu, J. B. Du, and I. Lin, J. Phys. D 27, 296 (1994).

${ }^{5}$ V. I. Molotkov, A. P. Nefedov, V. M. Torchinskii et al., Sov. Phys. JETP 89, 477 (1999); V. E. Fortov, A. G. Kharpak, S. A. Kharpak et al., Phys. Plasmas 7, 1374 (2000).

${ }^{6}$ A. Barken, N. D’Angelo, and R. L. Merlino, Planet. Space Sci. 44, 239 (1996).

${ }^{7}$ Y. Nakamura, H. Bailumg, and P. K. Shukla, Phys. Rev. Lett. 83, 1602 (1999).

${ }^{8}$ G. E. Morfill, H. M. Thomas, and M. Zuzic, in Advances in Dusty Plasmas, edited by P. K. Shukla, D. A. Mendis, and T. Desai (World Scientific, Singapore, 1997), pp. 99-142.

${ }^{9}$ R. L. Merlino, A. Barken, C. Thompson, and N. D'Angelo, Phys. Plasmas 5, 1607 (1998).

${ }^{10}$ M. Salimullah, Phys. Lett. A 215, 296 (1996).

${ }^{11}$ M. Salimullah and G. E. Morfill, Phys. Rev. E 59, R2558 (1999).

${ }^{12}$ N. D’ Angelo, Phys. Lett. A 292, 195 (2001).

${ }^{13}$ M. K. Islam, M. Salahuddin, A. K. Banerjee, and M. Salimullah, Phys. Plasmas 9, 2971 (2002). 